\title{
Synthesis and Cytotoxicity of POSS Modified Single Walled Carbon Nanotubes
}

\author{
Yuhua Xue and Hao Chen \\ Institute of Advanced Materials for Nano-Bio Applications, School of Ophthalmology \& Optometry, \\ Wenzhou Medical University, 270 Xueyuan Xi Road, Wenzhou, Zhejiang 325027, China \\ Correspondence should be addressed to Yuhua Xue; yuhua_xue@hotmail.com and Hao Chen; chenhao@mail.eye.ac.cn
}

Received 4 December 2014; Revised 24 January 2015; Accepted 3 February 2015

Academic Editor: Andrew R. Barron

Copyright (c) 2015 Y. Xue and H. Chen. This is an open access article distributed under the Creative Commons Attribution License, which permits unrestricted use, distribution, and reproduction in any medium, provided the original work is properly cited.

Single walled carbon nanotubes (SWNTs) decorated with polyhedral oligomeric silsesquioxane (POSS) were synthesized via the amide linkages between the acid treated SWNTs and amine-functionalized POSS. The successful modification of SWNTs with POSS was confirmed by Fourier transform infrared spectroscopy (FTIR), X-ray photoelectron spectroscopy (XPS), and UV-Vis spectra. The resulting SWNTs-POSS can be dispersed in both water and organic solutions. The biocompatibility and cytotoxicity of the SWNTs and SWNTs-POSS were evaluated by CCK-8 viability assays, which indicated that SWNTs-POSS exhibit very extremely low toxicity. The low toxicity of the POSS modified SWNTs leads to more opportunities for using carbon nanotubes in biomedical fields.

\section{Introduction}

Carbon nanotubes (CNTs) have drawn considerable attention for many years due to their excellent electrical, mechanical, thermal, and optical properties. The unique structure and excellent properties allow carbon nanotubes to be suitable for many applications [1-6], such as catalysts [2], dry adhesives [3], sensors [4], and devices [5]. Recently, carbon nanotubes have shown promising applications in biomedical systems [712]. For instance, carbon nanotubes can be used for imaging $[8,10]$, drug delivery [9], tissue engineering $[12,13]$, and so on. The wide usage of carbon nanotubes in biomedical systems and human daily life raises urgent questions about the safety of its use and toxicity assessment in various systems.

The biocompatibility and toxicity of carbon nanotubes have been researched in some medical fields. Recent studies have shown that the toxicity of CNTs is determined by their morphology, number of the walls, and functional groups. Lam et al. [14] found that single walled carbon nanotubes are more toxic than carbon black in lungs, and SWNTs can induce dose-dependent epithelioid granulomas and interstitial inflammation in the animals. When carbon nanotubes were induced into the abdominal cavity of mice, they would result in asbestos-like, pathogenic behavior [15]. Manna et al. [16] found that SWNTs can induce oxidative stress and activate nuclear transcription factor- $\mathrm{kB}$ in human keratinocytes. Bai et al. [17] found that repeated administrations of carbon nanotubes in male mice can cause reversible testis damage without affecting fertility. Recent studies show that surface modification can decrease the cytotoxicity of carbon nanotubes $[18,19]$.

Polyhedral oligomeric silsesquioxane (POSS), a welldefined organic/inorganic hybrid molecule, has received much interest due to its unique cage-like structure and high performance when combining with polymers. Our previous work has shown that POSS modified polymethylmethacrylate (PMMA) exhibited superhydrophobic properties [20] and POSS modified $\mathrm{Fe}_{3} \mathrm{O}_{4}$ nanoparticles showed both superhydrophobic and magnetic properties [21]. POSS modified graphene has also shown amazing properties for multiple applications [22]. As POSS is constituted by $\mathrm{Si}-\mathrm{O}$ and $\mathrm{Si}-$ $\mathrm{C}$ bonds, which is similar to the low toxic silicone, POSS is thought of as a potential building block for biomaterials [2326]. 


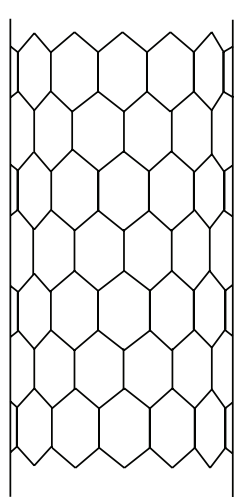

SWNTs
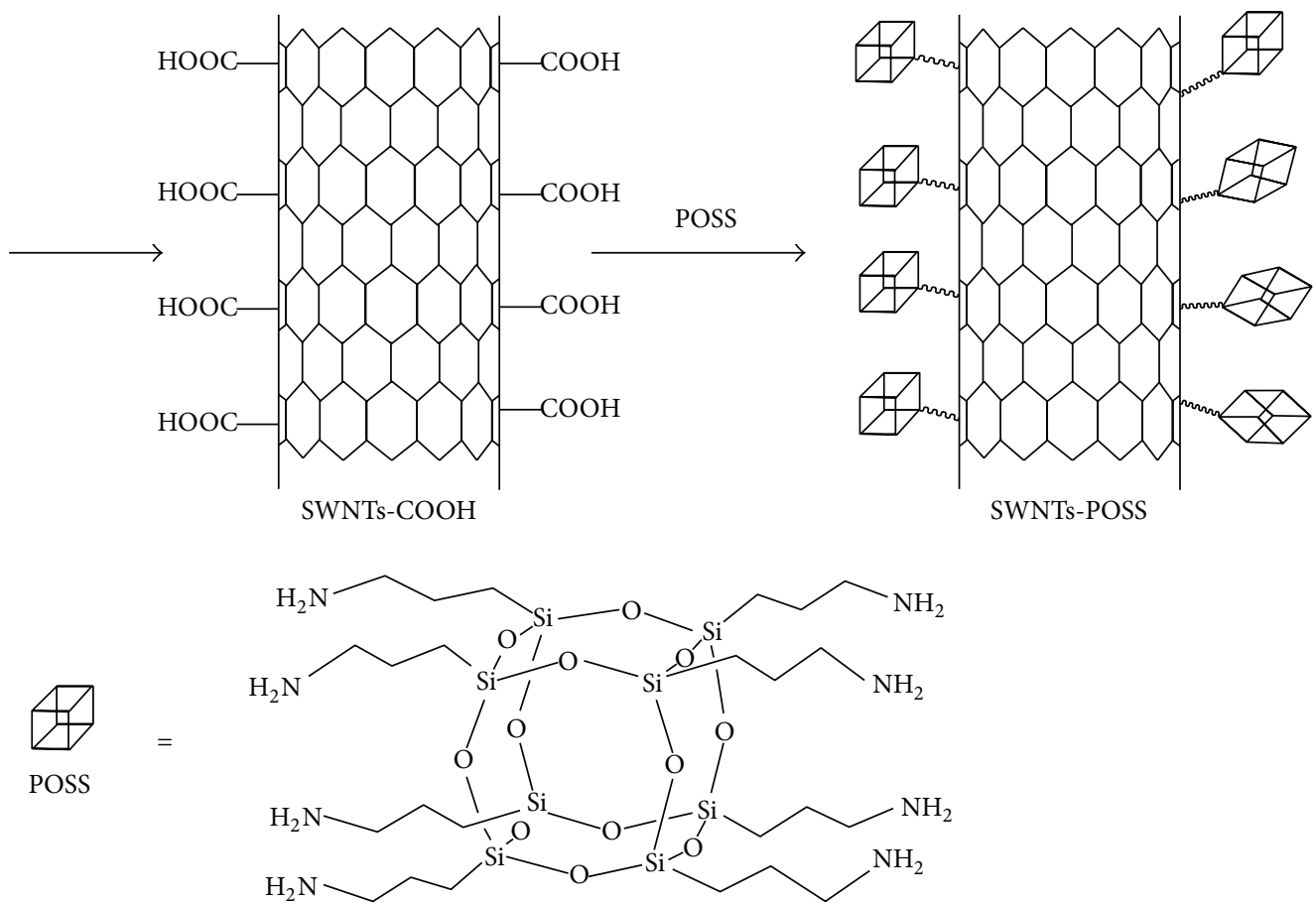

FIGURE 1: Schematic representation of the preparation route to SWNTs-POSS.

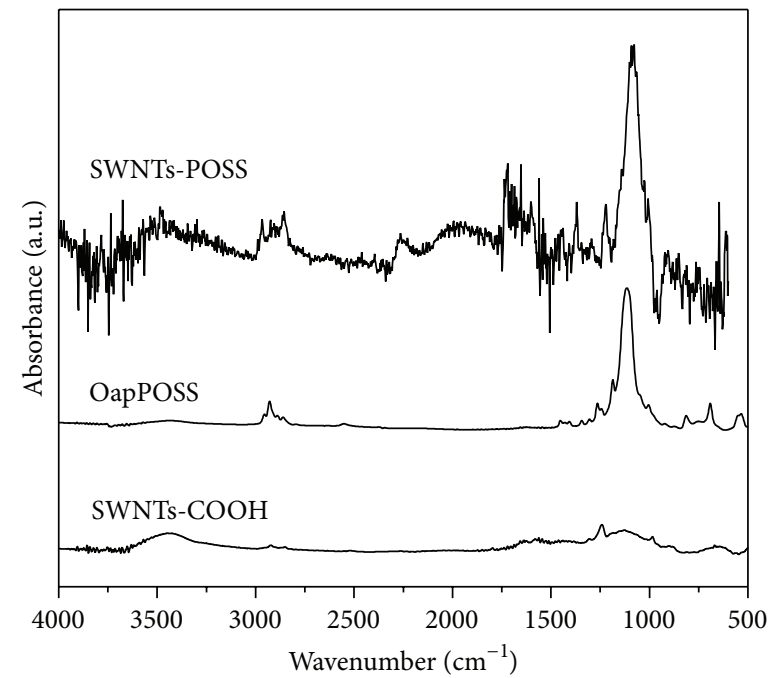

(a)

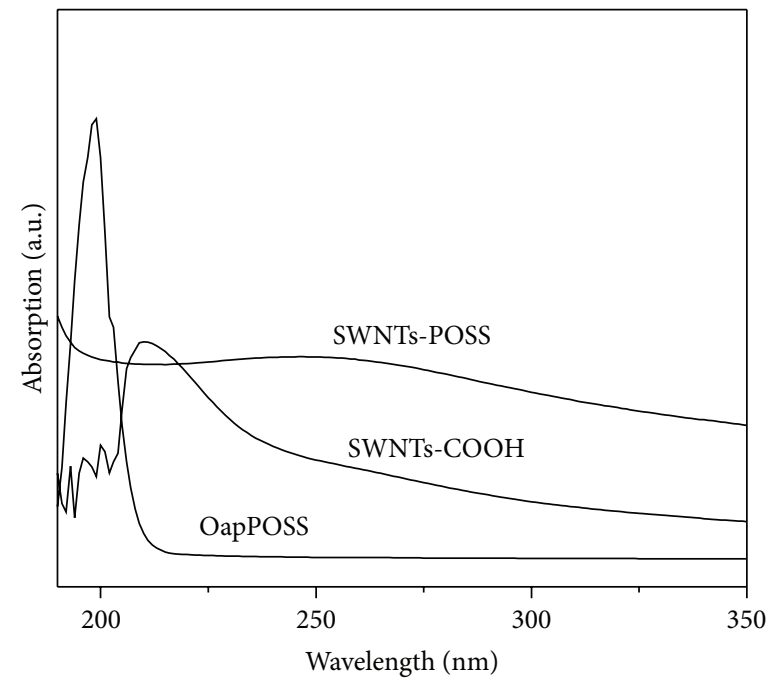

(b)

FIGURE 2: (a) FTIR spectra of OapPOSS, SWNTs-COOH, and SWNTs-POSS. (b) UV absorption spectra of OapPOSS, SWNTs-COOH, and SWNTs-POSS in water solution.

In this paper, we decorated single walled carbon nanotubes (SWNTs) with POSS via covalent bond. The cytotoxicity of POSS, SWNT, and POSS modified SWNTs on human retinal pigment epithelial (RPE) cells was assessed by using CCK-8 methods. POSS modified SWNTs shows extremely low cytotoxicity, which may greatly enlarge applications of carbon nanotubes in biomedical field.

\section{Materials and Methods}

2.1. Synthesis of Octaaminopropyl Polyhedral Oligomeric Silsesquioxane (OapPOSS). The OapPOSS was synthesized according to the literature method [27]. Typically, to a flask equipped with a magnetic stirrer, $360 \mathrm{~mL}$ of methanol, $27 \mathrm{~mL}$ of concentrated hydrochloric acid (37\%), and $15 \mathrm{~mL}$ 


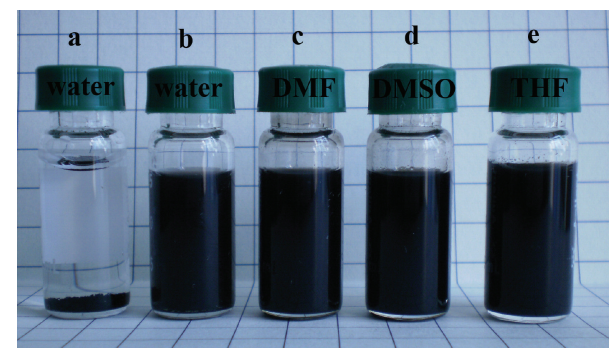

FIgURE 3: Photos of (a) SWNTs dispersed in water and (b-e) SWNTs-POSS dispersed in water and organic liquids.

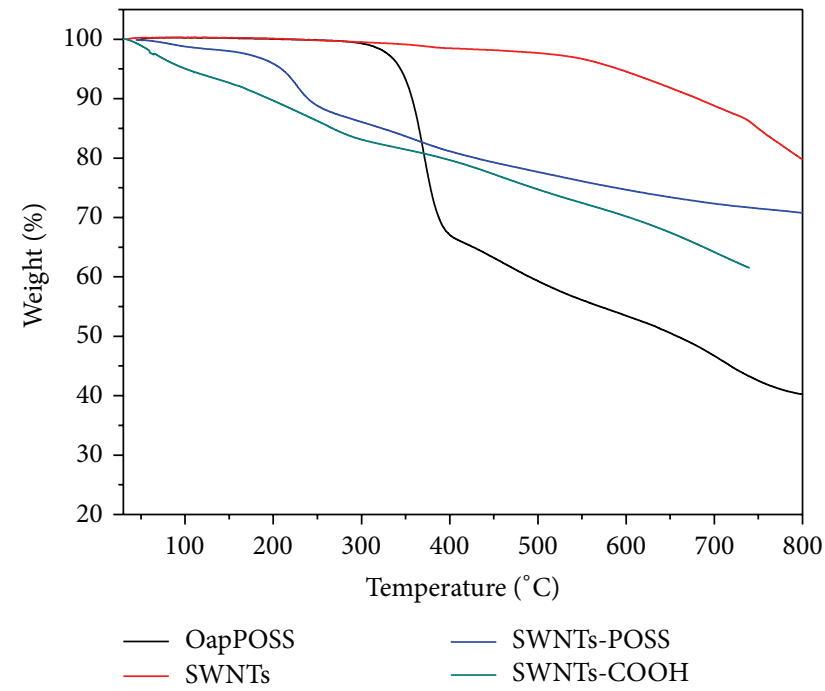

FIgURE 4: TGA curves of OapPOSS, SWNTs, SWNTs-POSS, and SWNTs-COOH.

( $0.0838 \mathrm{~mol})$ of $\gamma$-aminopropyltriethoxysilane were charged. The hydrolysis and rearrangement reaction was carried out for 6 weeks at room temperature and the microcrystalline precipitates were obtained. The product was collected $(3.7 \mathrm{~g}$, $30 \%$ yield) after filtration, washed with methanol, and dried in a vacuum oven. FTIR $\left(\mathrm{cm}^{-1}\right): 3200-2800(\mathrm{~N}-\mathrm{H}), 3000-$ $2800(\mathrm{C}-\mathrm{H}), 1105$ ( $\mathrm{Si}-\mathrm{O}-\mathrm{Si}) ;{ }^{29} \mathrm{Si} \mathrm{NMR}$ (ppm): 67.0 (s).

2.2. Synthesis of SWNTs-COOH. SWNTs were purchased from Nanjing XFNANO materials Tech Co., Ltd., China. $50 \mathrm{mg}$ of SWNTs was added into a $100 \mathrm{~mL}$ flask containing a mixture of $\mathrm{H}_{2} \mathrm{SO}_{4}(30 \mathrm{~mL}, 98 \%)$ and $\mathrm{HNO}_{3}(10 \mathrm{~mL}, 60 \%)$. The mixture was then ultrasonicated for 8 hours. The resulting solution was diluted with $200 \mathrm{~mL}$ of deionized water, followed by a vacuum-filtering through a $0.22 \mu \mathrm{m}$ polycarbonate film. The solid product was washed with deionized water three times. SWNTs functionalized with carboxyl groups were obtained after vacuum drying.

2.3. Preparation of SWNTs-POSS. In a $100 \mathrm{~mL}$ round bottom flask, $50 \mathrm{mg}$ of SWNTs-COOH, $2 \mathrm{~g}$ of OapPOSS, and $50 \mathrm{mg}$ of dicyclohexylcarbodiimide (DCC) (as catalyst) were dispersed

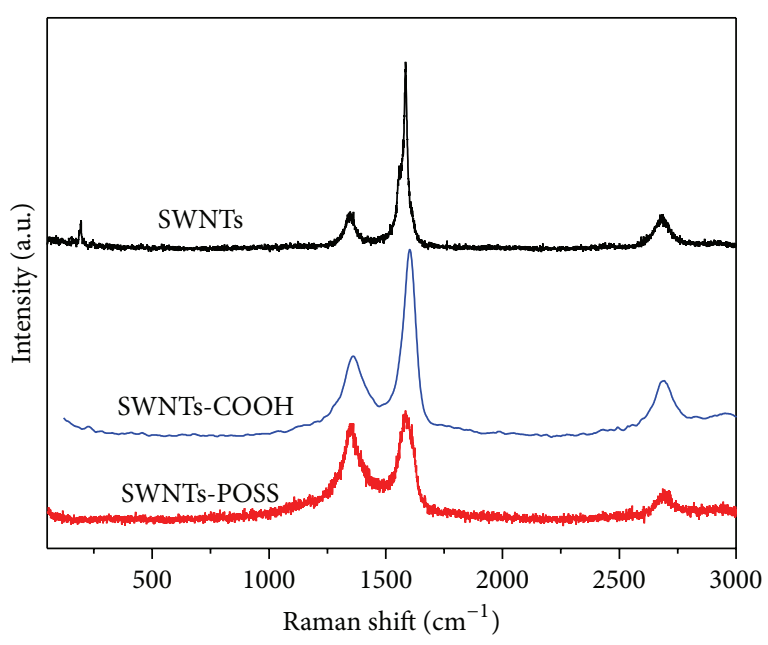

FIGURE 5: Raman spectra of SWNTs, SWNTs-COOH, and SWNTsPOSS.

in $40 \mathrm{~mL}$ ethanol, followed by ultrasonication for 10 minutes. The mixture was then refluxed for 24 hours at the temperature of $100^{\circ} \mathrm{C}$. When the reaction finished, the resulting solution was cooled down to room temperature and then vacuum filtered through a $0.22 \mu \mathrm{m}$ polycarbonate film. The solid product was thoroughly washed with distilled water for three times; then the product was washed with chloroform and dried in vacuum oven.

2.4. Characterization. Fourier transform infrared spectroscopy (FTIR) spectra were recorded on a PerkinElmer spectrum GX FTIR system at room temperature. The samples and $\mathrm{KBr}$ crystal were ground together using a mortar and pestle. The resulting ground power was pressed into small flakes for FTIR measurement. UV-Vis spectra were collected on an Agilent Cary 100 UV-Vis spectrophotometer; all the samples were dissolved in water solution for UV-Vis measurements. The thermogravimetric analysis was taken on a TA instrument with a heating rate of $10^{\circ} \mathrm{C}$. The Raman measurement was carried out on a Raman spectroscopy (Renishaw) using $514 \mathrm{~nm}$ laser. X-ray photoelectron spectroscopic (XPS) spectra were recorded on a PHI 5000 VersaProbe.

\section{Results and Discussion}

Figure 1 illustrates the synthesis process of POSS functionalized SWNTs. Firstly, SWNTs were converted into SWNTs$\mathrm{COOH}$ by surface modifying SWNTs with a mixture of $\mathrm{H}_{2} \mathrm{SO}_{4}$ and $\mathrm{HNO}_{3}$. POSS was then incorporated into SWNT via covalent bond between the amine groups in POSS and acid groups in SWNTs-COOH.

The structure of the as-prepared SWNTs-POSS was characterized by FTIR and UV. Figure 2(a) shows the FTIR spectra of SWNTs, OapPOSS, and SWNTs-POSS. The spectrum of OapPOSS shows a very sharp peak at $1110 \mathrm{~cm}^{-1}$, which is corresponding to the $\mathrm{Si}-\mathrm{O}-\mathrm{Si}$ stretching band. This peak was also found in the spectrum of SWNTsPOSS, indicating POSS has been incorporated into SWNTs. 


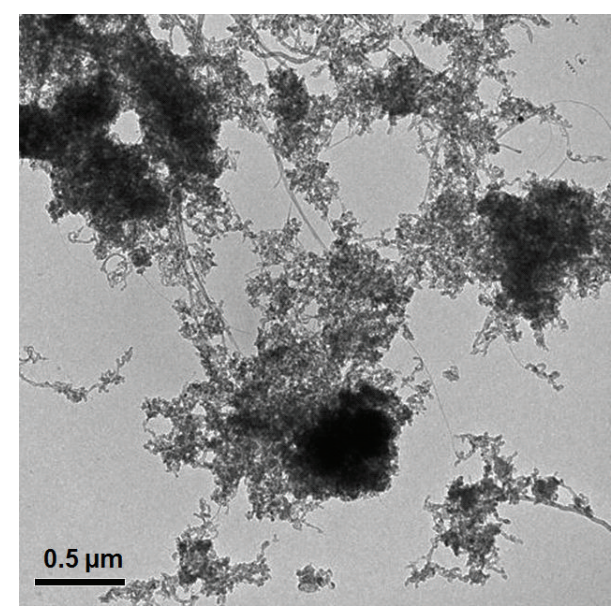

(a)

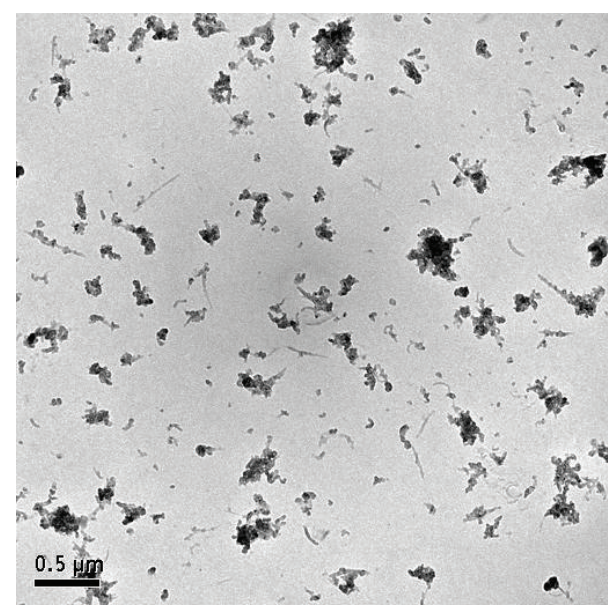

(b)

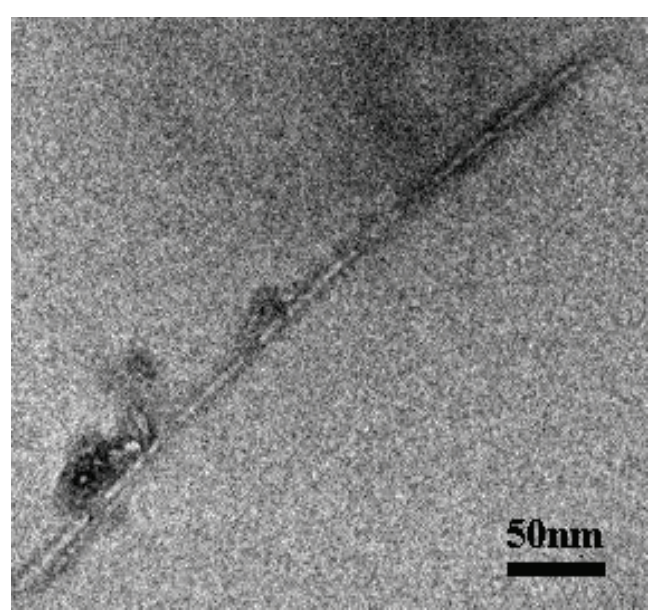

(c)

FIGURE 6: TEM images of (a) SWNTs and (b and c) SWNTs-POSS.

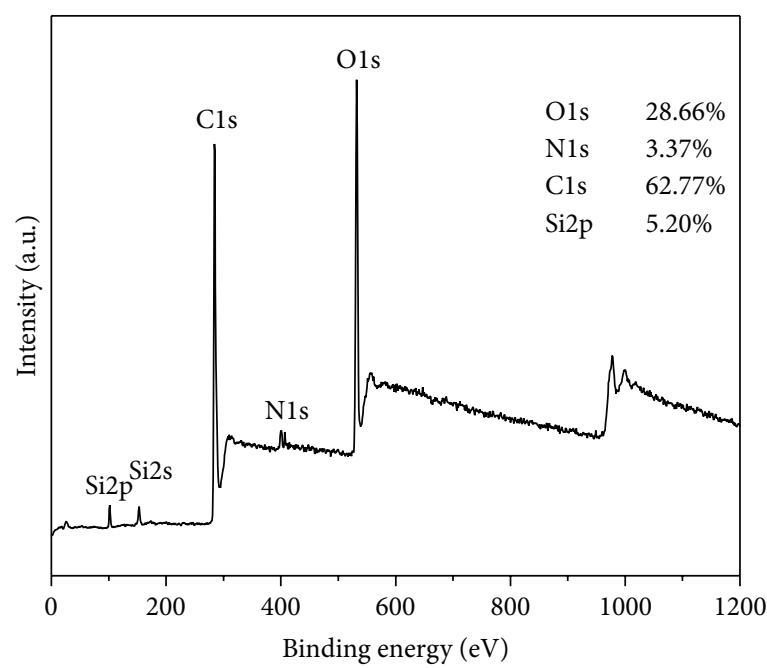

Figure 7: XPS spectrum of SWNTs-POSS. 


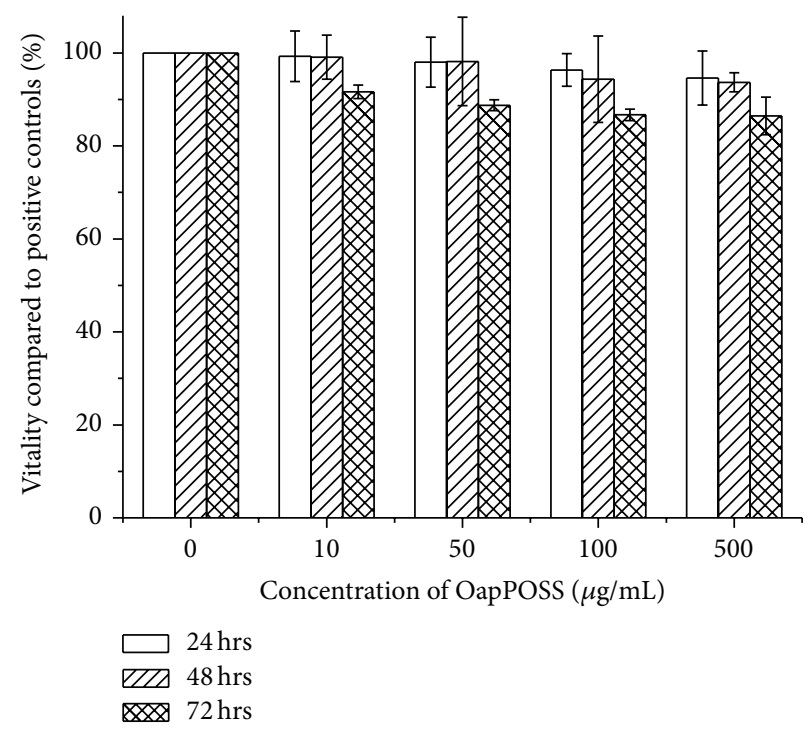

FIGURE 8: Vitality of RPE cell incubated with OapPOSS at 24, 48, and 72 hours.

The synthetic results also characterized UV absorption spectra. As is shown in Figure 2(b), the spectrum of OapPOSS has a strong absorption peak at $200 \mathrm{~nm}$, while SWNTs$\mathrm{COOH}$ has a broad absorption band at $225 \mathrm{~nm}$. After being modified with POSS, the resulting SWNTs-POSS have a broad adsorption peak at about $250 \mathrm{~nm}$.

Figure 3 shows the photographs of SWNTs and SWNTsPOSS dispersing in water and organic solutions. On dispersing the neat SWNTs in water, the black powder quickly deposited at the bottom of the vial. However, when SWNTs were surface modified with OapPOSS, the resulting SWNTsPOSS can be stably dispersed not only in water, but also in organic solutions, such as N,N-dimethylformamide (DMF), dimethyl sulfoxide (DMSO), and tetrahydrofuran (THF).

The thermal stability of OapPOSS, SWNTs, SWNTs$\mathrm{COOH}$, and SWNTs-POSS was studied by TGA. As is presented in Figure 4, the pristine SWNTs have very high thermal stability and the initial decomposition temperature (Td) is as high as $600^{\circ} \mathrm{C}$, while OapPOSs has a Td at $347^{\circ} \mathrm{C}$. The char yields of SWNTs and OapPOSS at $800^{\circ} \mathrm{C}$ are $79.71 \%$ and $40.23 \%$, respectively. The initial decomposition temperatures of SWNTs-POSS and SWNTs-COOH are greatly decreased when comparing with pristine SWNTs because of the functional groups of POSS and $\mathrm{COOH}$. The thermal stability of SWNTs-POSS was better than SWNTs-COOH, because the unstable $\mathrm{COOH}$ groups in SWNTs-COOH were reacted with $\mathrm{NH}_{2}$ groups in POSS. TGA results also confirmed that OapPOSS has been successfully incorporated into SWNTs.

Figure 5 is the Raman results of SWNTs, SWNTs-COOH, and SWNTs-POSS. In the Raman spectrum of SWNTs, there is a small peak at $195 \mathrm{~cm}^{-1}$, which is the characteristic peak of SWNTs. However, this peak disappears in the Raman spectra of both SWNTs-COOH and SWNTs-POSS. Moreover, the ratio of D band to G band of SWNTs-POSS is much higher than that of SWNTs and SWNTs-COOH, because the structure of SWNTs was destroyed during the acids treatment and POSS functionalization process. All these have indicated that POSS has been successfully synthesized onto SWNTs' surface.

Figure 6 shows TEM images of SWNTs and SWNTsPOSS with different magnifications. As is shown in Figure 6(a), the SWNTs were aggregated together. After being treated with acid and reacted with POSS, SWNTs-POSS become short and separated (Figure 6(b)). In Figure 6(c), some black dots, which were though as POSS, were found on the surface of carbon nanotubes. X-ray photoelectron spectroscopy (XPS) has also been used to characterize the SWNTs-POSS. As is shown in Figure 7, the atom percent of the carbon, oxygen, silicon, and nitrogen is $62.77 \%, 28.66 \%$, $5.20 \%$, and $3.37 \%$, respectively. The appearance of silicon and nitrogen peaks in XPS curve of SWNTs-POSS (Figure 7) came from POSS, which can also confirm that SWNTs-POSS were synthesized.

The cytotoxicity and biocompatibility of carbon nanotubes are very important when one introduces these materials into biomedical applications. OapPOSS was used to modify the surface of SWNTs in order to designing biocompatible nanohybrids. CCK-8 viability assays were used to assess the cytotoxicity of OapPOSS on human retinal pigment epithelial (RPE) cells firstly. As is shown in Figure 8, the cell survival is more than $85 \%$ even at the high concentration $(500 \mu \mathrm{g} / \mathrm{mL})$ of OapPOSS after 72 hours. OapPOSS demonstrates extremely low toxicity levels.

When combining POSS with SWNTs, POSS formed a protective layer surrounding carbon nanotubes. As is presented in Figure 9, The RPE cell viability decreased with increasing the concentration of SWNTs, SWNTs-COOH, and 


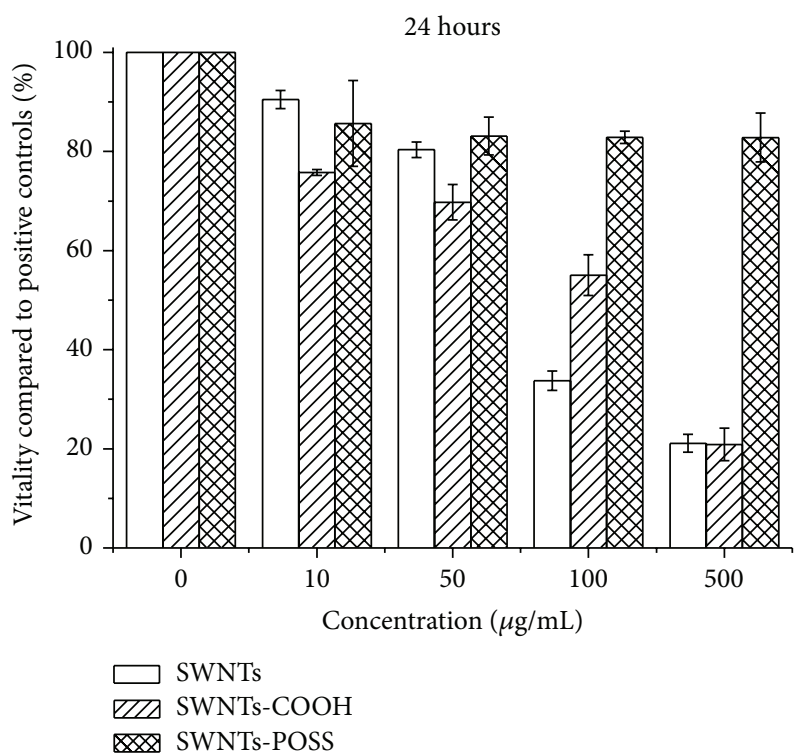

(a)

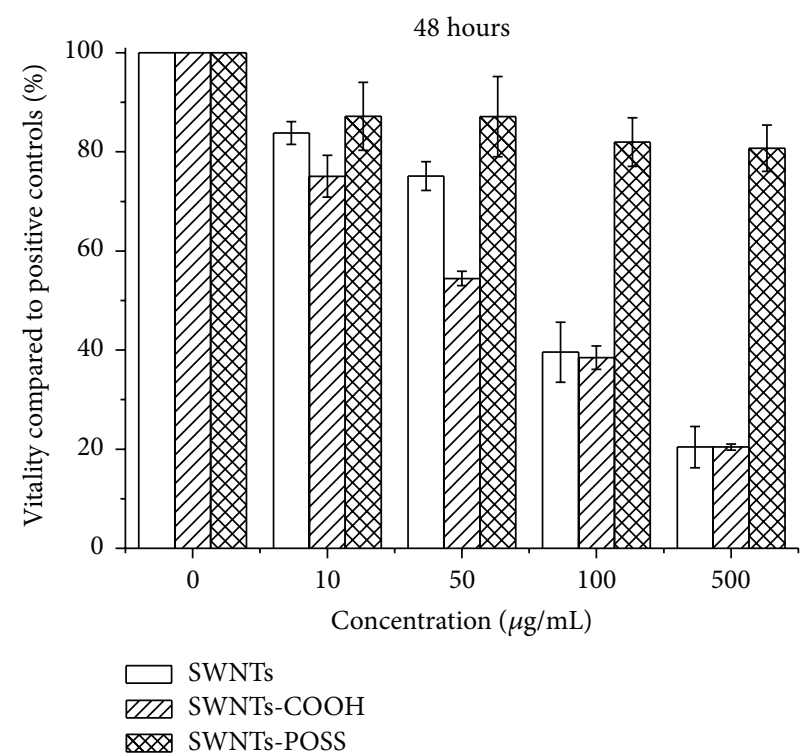

(b)

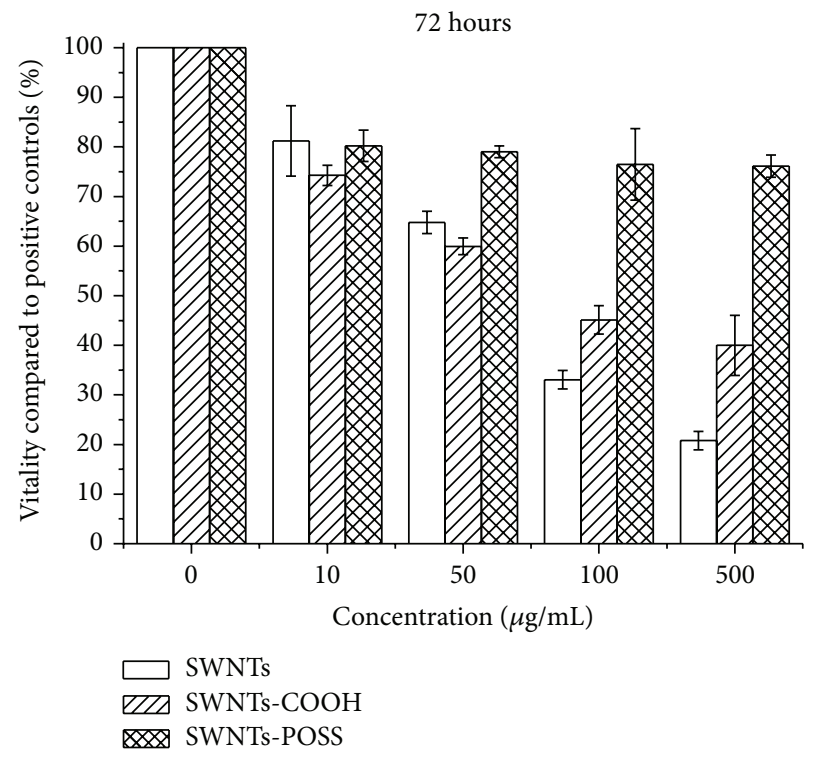

(c)

Figure 9: Vitality of RPE cell incubated with SWNTs, SWNTs-COOH, and SWNTs-POSS at (a) 24 hours, (b) 48 hours, and (c) 72 hours.

SWNTs-POSS. However, the highest cell viability was found in those culture solutions with SWNTs-POSS, which indicated that the cytotoxicity of SWNTs was greatly decreased when being functionalized with OapPOSS.

\section{Conclusion}

In summary, we have successfully incorporated POSS onto the surface of single walled carbon nanotubes via covalent bond. The results from FTIR, XPS, UV, and TGA clearly indicated that SWNTs-POSS were obtained successfully. CCK-8 assays demonstrate that OapPOSS has very low cytotoxicity and high biocompatibility. When combining OapPOSS and SWNTs, POSS formed a protective layer surrounding carbon nanotubes, and the cytotoxicity was greatly decreased. It will greatly extend the biomedical applications of carbon nanotubes.

\section{Conflict of Interests}

The authors declare that there is no conflict of interests regarding the publication of this paper. 


\section{Acknowledgment}

This work was supported financially by NSFC (51202167 and 81271703).

\section{References}

[1] M. Xu, D. N. Futaba, T. Yamada, M. Yumura, and K. Hata, "Carbon nanotubes with temperature-invariant viscoelasticity from $-196^{\circ}$ to $1000^{\circ}$ C," Science, vol. 330, no. 6009, pp. 1364-1368, 2010.

[2] K. Gong, F. Du, Z. Xia, M. Durstock, and L. Dai, "Nitrogendoped carbon nanotube arrays with high electrocatalytic activity for oxygen reduction," Science, vol. 323, no. 5915, pp. 760764,2009 .

[3] L. Qu, L. Dai, M. Stone, Z. Xia, and Z. L. Wang, "Carbon nanotube arrays with strong shear binding-on and easy normal lifting-off," Science, vol. 322, no. 5899, pp. 238-242, 2008.

[4] P. Diao and Z. Liu, "Vertically aligned single-walled carbon nanotubes by chemical assembly-methodology, properties, and applications," Advanced Materials, vol. 22, no. 13, pp. 14301449, 2010.

[5] K. T. Constantopoulos, C. J. Shearer, A. V. Ellis, N. H. Voelcker, and J. G. Shapter, "Carbon nanotubes anchored to silicon for device fabrication," Advanced Materials, vol. 22, no. 5, pp. 557571, 2010.

[6] J. Yao, Z. Jin, L. Zhong, D. Natelson, and J. M. Tour, “Twoterminal nonvolatile memories based on single-walled carbon nanotubes," ACS Nano, vol. 3, no. 12, pp. 4122-4126, 2009.

[7] Z. Liu, S. M. Tabakman, Z. Chen, and H. Dai, "Preparation of carbon nanotube bioconjugates for biomedical applications," Nature Protocols, vol. 4, no. 9, pp. 1372-1382, 2009.

[8] K. Welsher, Z. Liu, S. P. Sherlock et al., "A route to brightly fluorescent carbon nanotubes for near-infrared imaging in mice," Nature Nanotechnology, vol. 4, no. 11, pp. 773-780, 2009.

[9] S. Dhar, Z. Liu, J. Thomale, H. Dai, and S. J. Lippard, “Targeted single-wall carbon nanotube-mediated $\mathrm{Pt}(\mathrm{IV})$ prodrug delivery using folate as a homing device," Journal of the American Chemical Society, vol. 130, no. 34, pp. 11467-11476, 2008.

[10] A. De La Zerda, C. Zavaleta, S. Keren et al., "Carbon nanotubes as photoacoustic molecular imaging agents in living mice," Nature Nanotechnology, vol. 3, no. 9, pp. 557-562, 2008.

[11] Z. Liu, W. B. Cai, L. N. He et al., "In vivo biodistribution and highly efficient tumour targeting of carbon nanotubes in mice," Nature Nanotechnology, vol. 2, no. 1, pp. 47-52, 2007.

[12] B. S. Harrison and A. Atala, "Carbon nanotube applications for tissue engineering," Biomaterials, vol. 28, no. 2, pp. 344-353, 2007.

[13] L. Lacerda, H. Ali-Boucetta, M. A. Herrero et al., "Tissue histology and physiology following intravenous administration of different types of functionalized multiwalled carbon nanotubes," Nanomedicine, vol. 3, no. 2, pp. 149-161, 2008.

[14] C.-W. Lam, J. T. James, R. McCluskey, and R. L. Hunter, "Pulmonary toxicity of single-wall carbon nanotubes in mice 7 and 90 days after intractracheal instillation," Toxicological Sciences, vol. 77, no. 1, pp. 126-134, 2004.

[15] C. A. Poland, R. Duffin, I. Kinloch et al., "Carbon nanotubes introduced into the abdominal cavity of mice show asbestoslike pathogenicity in a pilot study," Nature Nanotechnology, vol. 3, no. 7, pp. 423-428, 2008.
[16] S. K. Manna, S. Sarkar, J. Barr et al., "Single-walled carbon nanotube induces oxidative stress and activates nuclear transcription factor- $\kappa \mathrm{B}$ in human keratinocytes," Nano Letters, vol. 5, no. 9, pp. 1676-1684, 2005.

[17] Y. Bai, Y. Zhang, J. P. Zhang et al., "Repeated administrations of carbon nanotubes in male mice cause reversible testis damage without affecting fertility," Nature Nanotechnology, vol. 5, no. 9, pp. 683-689, 2010.

[18] K. Kostarelos, "The long and short of carbon nanotube toxicity," Nature Biotechnology, vol. 26, no. 7, pp. 774-776, 2008.

[19] M. L. Schipper, N. Nakayama-Ratchford, C. R. Davis et al., "A pilot toxicology study of single-walled carbon nanotubes in a small sample of mice," Nature Nanotechnology, vol. 3, no. 4, pp. 216-221, 2008.

[20] Y. H. Xue, H. X. Wang, D. S. Yu et al., "Superhydrophobic electrospun POSS-PMMA copolymer fibres with highly ordered nanofibrillar and surface structures," Chemical Communications, no. 42, pp. 6418-6420, 2009.

[21] Y. H. Xue, H. X. Wang, Y. Zhao et al., "Magnetic liquid marbles: a 'precise' miniature reactor," Advanced Materials, vol. 22, no. 43, pp. 4814-4818, 2010.

[22] Y. Xue, Y. Liu, F. Lu, J. Qu, H. Chen, and L. Dai, "Functionalization of graphene oxide with polyhedral oligomeric silsesquioxane (POSS) for multifunctional applications," The Journal of Physical Chemistry Letters, vol. 3, no. 12, pp. 1607-1612, 2012.

[23] J. Wu and P. T. Mather, "POSS polymers: physical properties and biomaterials applications," Polymer Reviews, vol. 49, no. 1, pp. 25-63, 2009.

[24] R. Y. Kannan, H. J. Salacinski, P. E. Butler, and A. M. Seifalian, "Polyhedral oligomeric silsesquioxane nanocomposites: the next generation material for biomedical applications," Accounts of Chemical Research, vol. 38, no. 11, pp. 879-884, 2005.

[25] D. B. Cordes, P. D. Lickiss, and F. Rataboul, "Recent developments in the chemistry of cubic polyhedral oligosilsesquioxanes," Chemical Reviews, vol. 110, no. 4, pp. 2081-2173, 2010.

[26] C. McCusker, J. B. Carroll, and V. M. Rotelo, "Cationic polyhedral oligomeric silsesquioxane (POSS) units as carriers for drug delivery processes," Chemical Communications, no. 8, pp. 996998, 2005.

[27] H. Liu, W. Zhang, and S. Zheng, "Montmorillonite intercalated by ammonium of octaaminopropyl polyhedral oligomeric silsesquioxane and its nanocomposites with epoxy resin," Polymer, vol. 46, no. 1, pp. 157-165, 2005. 

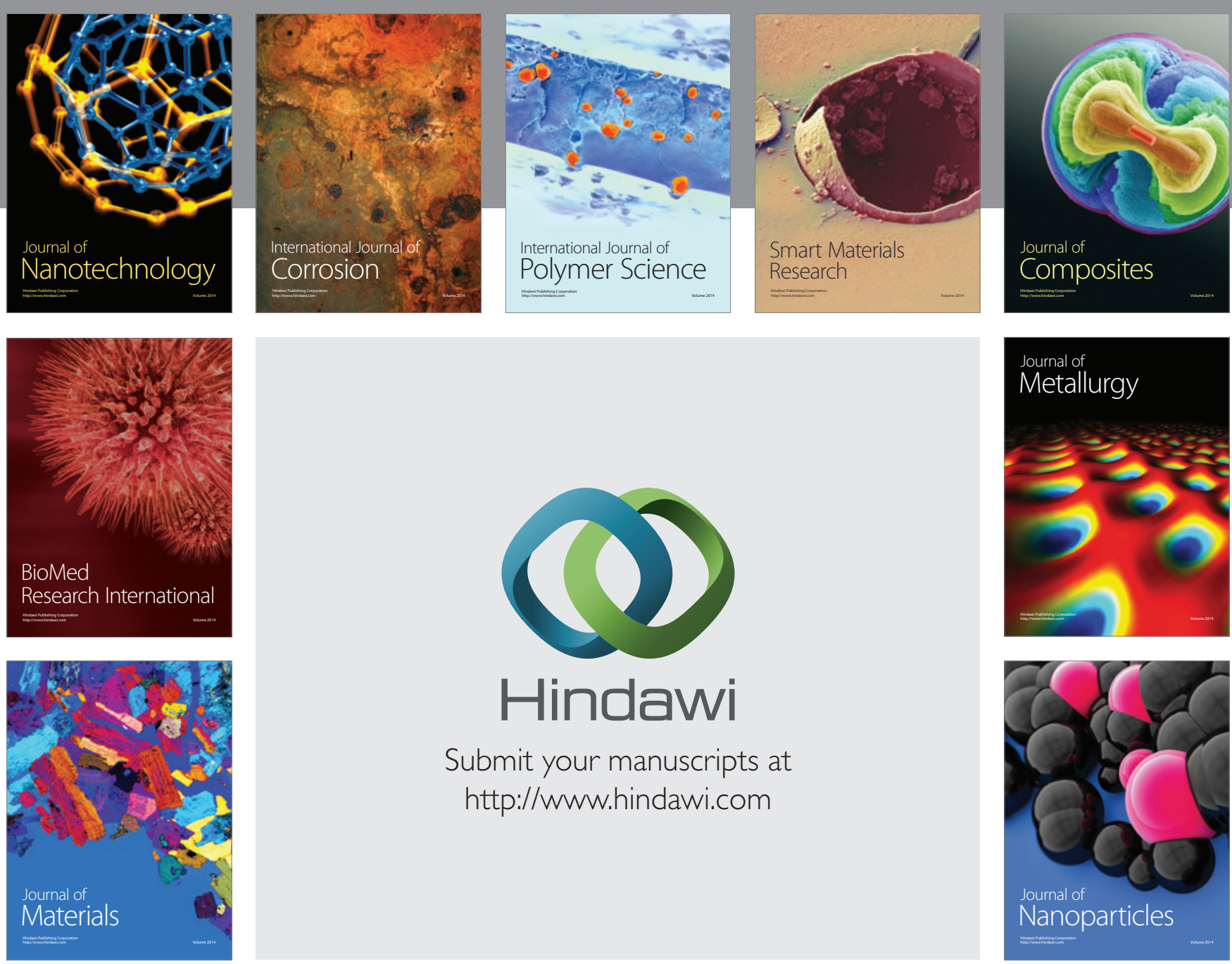

Submit your manuscripts at http://www.hindawi.com
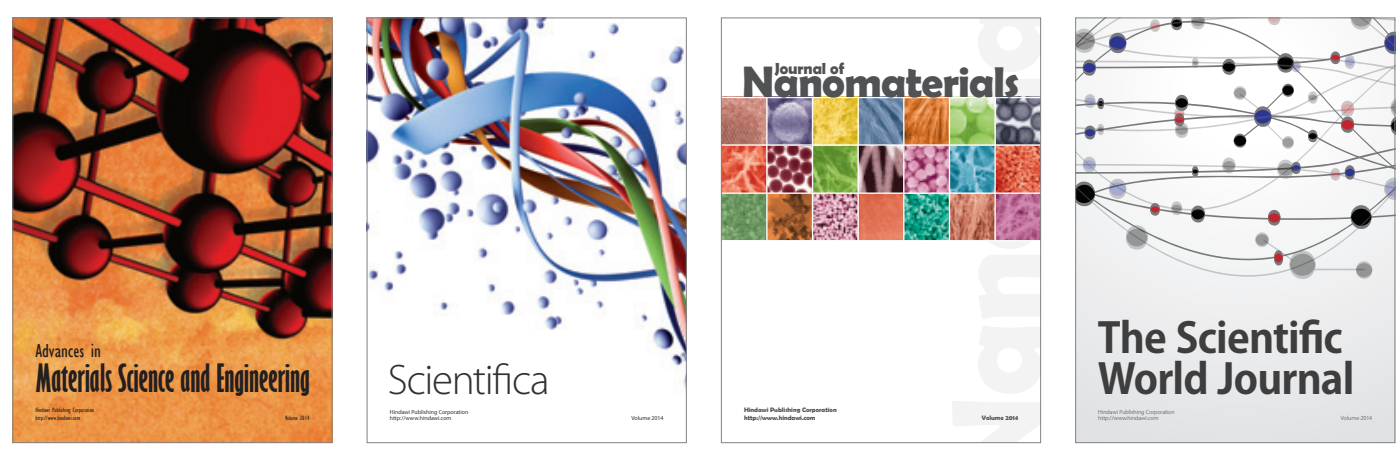

\section{The Scientific World Journal}
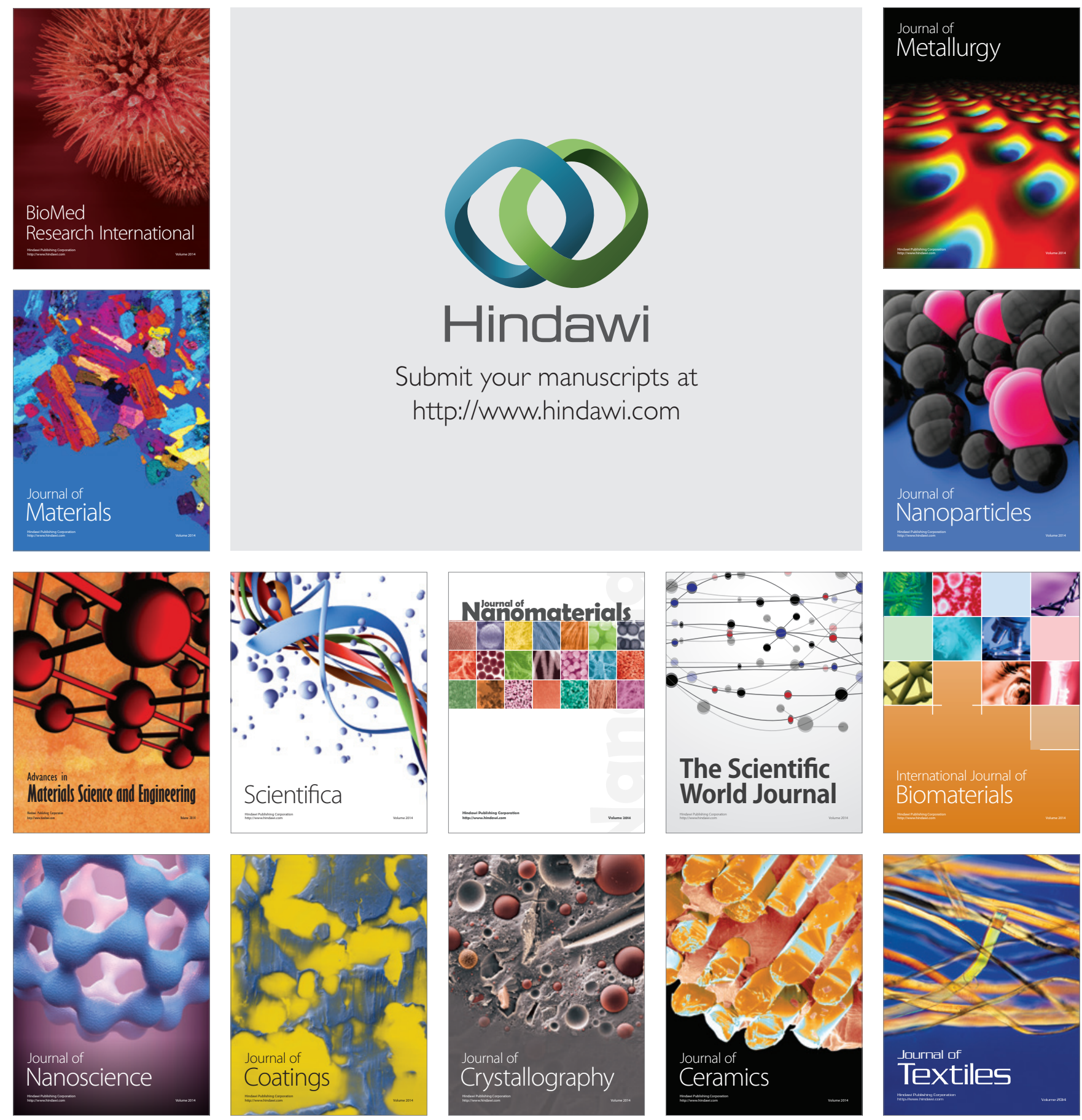